\title{
iPS cells to model CDKL5-related disorders
}

\author{
Mariangela Amenduni ${ }^{1,7}$, Roberta De Filippis ${ }^{1,7}$, Aaron YL Cheung ${ }^{2}$, Vittoria Disciglio ${ }^{1}$, \\ Maria Carmela Epistolato ${ }^{1}$, Francesca Ariani ${ }^{1}$, Francesca Mari ${ }^{1,3}$, Maria Antonietta Mencarelli ${ }^{1,3}$, \\ Youssef Hayek ${ }^{4}$, Alessandra Renieri ${ }^{\star, 1,3}$, James Ellis ${ }^{2,5,6}$ and Ilaria Meloni ${ }^{1}$
}

Rett syndrome (RTT) is a progressive neurologic disorder representing one of the most common causes of mental retardation in females. To date mutations in three genes have been associated with this condition. Classic RTT is caused by mutations in the MECP2 gene, whereas variants can be due to mutations in either MECP2 or FOXG1 or CDKL5. Mutations in CDKL5 have been identified both in females with the early onset seizure variant of RTT and in males with X-linked epileptic encephalopathy. CDKL5 is a kinase protein highly expressed in neurons, but its exact function inside the cell is unknown. To address this issue we established a human cellular model for CDKL5-related disease using the recently developed technology of induced pluripotent stem cells (iPSCs). iPSCs can be expanded indefinitely and differentiated in vitro into many different cell types, including neurons. These features make them the ideal tool to study disease mechanisms directly on the primarily affected neuronal cells. We derived iPSCs from fibroblasts of one female with p.Q347X and one male with p.T288I mutation, affected by early onset seizure variant and X-linked epileptic encephalopathy, respectively. We demonstrated that female CDKL5-mutated iPSCs maintain X-chromosome inactivation and clones express either the mutant $C D K L 5$ allele or the wild-type allele that serve as an ideal experimental control. Array CGH indicates normal isogenic molecular karyotypes without detection of de novo CNVs in the CDKL5-mutated iPSCs. Furthermore, the iPS cells can be differentiated into neurons and are thus suitable to model disease pathogenesis in vitro.

European Journal of Human Genetics (2011) 19, 1246-1255; doi:10.1038/ejhg.2011.131; published online 13 July 2011

Keywords: CDKL5; Rett syndrome; induced pluripotent stem cells (iPSC); disease modelling

\section{INTRODUCTION}

Rett syndrome (RTT) is a progressive neurological disorder that affects 1 in 10000 girls worldwide and represents one of the most common causes of mental retardation in females. RTT is characterized by an apparently normal development for the first 6-18 months of life, followed by regression with the onset of clinical signs including mental retardation, loss of speech, acquired microcephaly, growth retardation, autistic features, seizures, ataxia and hand stereotypies. ${ }^{1}$ Beside the classic form, several RTT variants have been described including the Zappella variant, the congenital form, the 'forme fruste' and the early onset seizures variant. ${ }^{2-5}$ In past years, mutations in three genes have been associated with classic and/or variant RTT: MECP2 and CDKL5, located on the X chromosome, and FOXG1, on chromosome 14. ${ }^{1,6,7}$ About $80 \%$ of classic RTT cases are caused by mutations in MECP2 that encodes for a methyl-CpG-binding protein involved in the regulation of gene expression. ${ }^{8,9}$ To investigate the molecular mechanisms leading from MECP2 mutations to RTT onset, different mouse models have been generated and extensively characterized. ${ }^{10-13}$ These models allowed identification of specific alterations in glutamatergic neurons: ${ }^{13}$ cells lacking Mecp2 have reduced synapse numbers and, accordingly, they show a reduced synaptic response. The opposite effects are elicited by Mecp2 over-expression. In spite of these important breakthroughs on the pathophysiology of RTT in animal models, the inaccessibility of the main affected human tissue and the impossibility of establishing good in vitro human cellular models have greatly complicated research efforts. In this respect, the development in 2007 of induced pluripotent stem cells (iPSCs) offered an unprecedented opportunity for the creation of patient- and disease-specific human cellular models. In fact, iPSCs can be obtained from differentiated somatic cells, for example fibroblasts, through the induction of expression of a few transcription factors related to pluripotency, usually OCT-4, SOX-2, c-MYC and KLF- $4 .{ }^{14}$ iPSCs are similar to human embryonic stem cells (hESCs) in morphology, proliferation, gene expression and in vitro differentiation potential. Like hESCs, they can be expanded indefinitely and differentiated in vitro into many different cell types. ${ }^{14}$ These features make them the ideal tool to study disease mechanisms directly on the primarily affected cells. Up to now, iPSCs have been successfully derived from patients with both neurodegenerative (ALS, SMA, Parkinson, HD, FD) and neurodevelopmental (FRAXA, PW-AS) disorders, and neuronal differentiation has been performed in some cases, using protocols developed for hESCs. ${ }^{15-17}$ Interestingly, a consistent neuronal phenotype was observed for SMA and FD, confirming the potential of this technology to model neurological disorders. ${ }^{15}$ Very recently, Marchetto et al ${ }^{18}$ applied this technology to model RTT in vitro. In fact they derived iPSCs from MECP2-mutated patients fibroblasts and differentiated them into glutamatergic neurons. They found that RTT neurons have a reduced number of dendritic spines compared with those derived from control

\footnotetext{
${ }^{1}$ Medical Genetics, Department of Biotechnology, University of Siena, Siena, Italy; ${ }^{2}$ Developmental \& Stem Cell Biology Program, SickKids, Toronto, Ontario, Canada; ${ }^{3}$ Medical Genetics, Azienda Ospedaliera Universitaria Senese, Siena, Italy; ${ }^{4}$ Child Neuropsychiatry, University hospital of Siena, Siena, Italy; ${ }^{5}$ Ontario Human iPS Cell Facility, SickKids, Toronto, Ontario, Canada; ${ }^{6}$ Department of Molecular Genetics, University of Toronto, Toronto, Ontario, Canada

*Correspondence: Professor A Renieri, Medical Genetics, Department of Biotechnology, University of Siena, Policlinico S. Maria alle Scotte, viale Bracci 2, 53100 Siena, Italy. Tel: +39 0577 233303; Fax: +39 0577 233325; E-mail: renieri@unisi.it

${ }^{7}$ These authors contributed equally to this work.

Received 31 December 2010; revised 15 April 2011; accepted 3 June 2011; published online 13 July 2011
} 
a

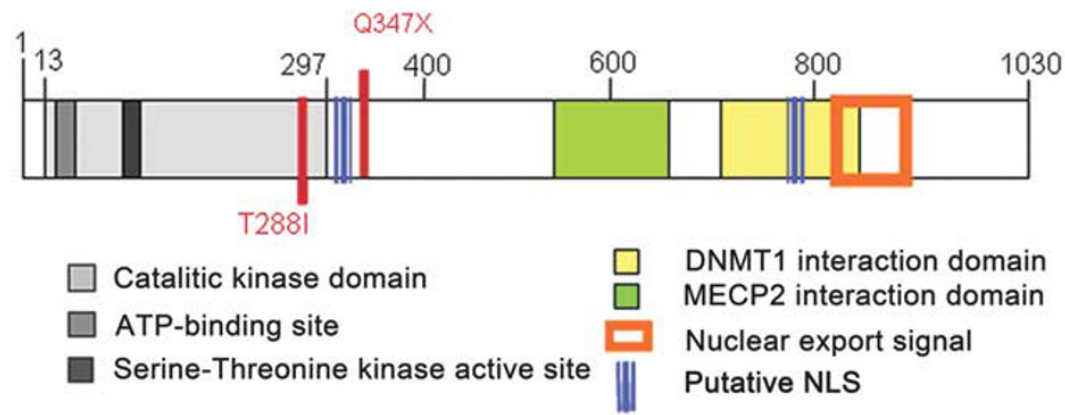

b
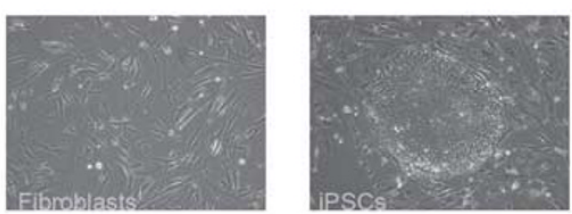

c

iPSC\#19

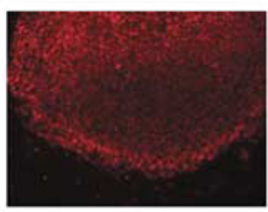

iPSC\#20

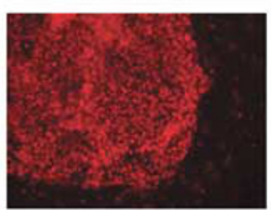

iPSC\#58

NANOG
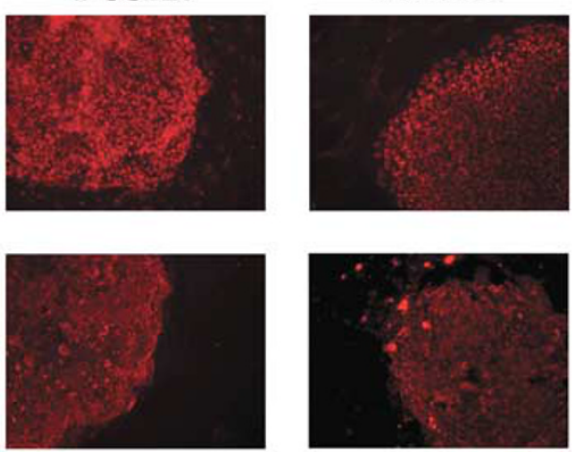

SSEA4
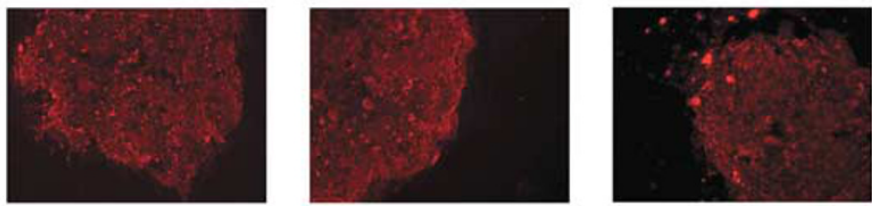

TRA-1-60
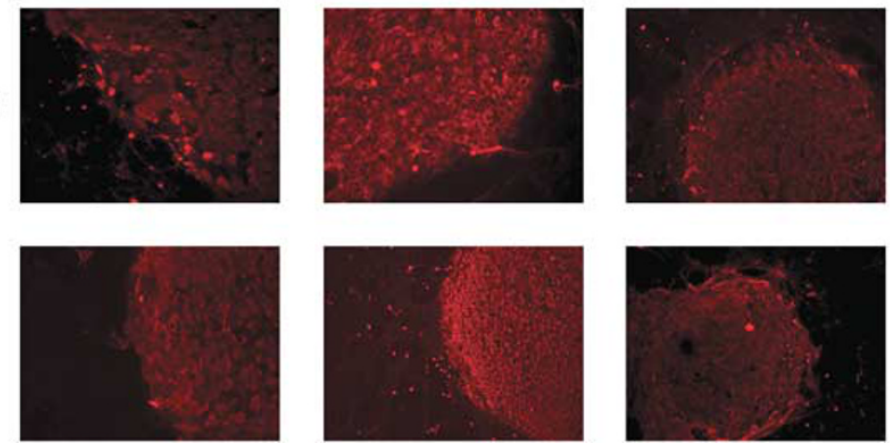

Figure 1 Generation and characterization of iPSCs. (a) Schematic representation of CDKL5 protein structure with the known functional domains. The mutations identified in the patients reported in this study are shown in red. (b) Phase contrast image of human fibroblasts morphology before reprogramming (left) and typical morphology of one iPSC clone (right). Cells surrounding the clone are mitomycin-C-inactivated mouse embryo fibroblasts (feeders). (c) Representative images of immunostaining for pluripotency markers for two clones derived from patient 1 (iPSC \#19 and iPSC \#20) and one clone from patient 2 (iPSC \#58). Images are at $\times 20$ magnification.

iPSCs or hESCs. Moreover, they also demonstrated an important reduction in the frequency and amplitude of spontaneous excitatory and inhibitory postsynaptic currents in RTT neurons. It is important to underline that these findings are in accordance with the data on mouse models and thus demonstrate the applicability of iPSCs technology to model RTT in vitro.

Unlike the classic form, RTT variants are mainly due to mutations in the other two genes, CDKL5 and FOXG1. In particular, mutations in CDKL5 have been identified in the early onset seizures variant of RTT in females and in X-linked epileptic encephalopathy in males. ${ }^{19,20}$ CDKL5 is a kinase protein that shuttles between the cytoplasm and the nucleus (Figure 1a). ${ }^{20} \mathrm{~A}$ direct interaction with $\mathrm{MeCP} 2$ has been demonstrated, in accordance with their common involvement in RTT: CDKL5 can bind and phosphorylate MeCP2 in vitro, and MeCP2 in turn regulates CDKL5 gene expression, at least in certain brain regions. ${ }^{19,21,22}$ Moreover, the two proteins bind to different regions of DNMT1 further suggesting the possibility of a participation in common pathways. ${ }^{23}$ Intriguingly, CDKL5 shows high expression levels in neurons but it is undetectable in glial cells, indicating an important role in neuronal development and/or function. ${ }^{20} \mathrm{~A}$ mouse model for CDKL5-related disease is currently lacking, limiting our knowledge of the function of CDKL5 inside the neuron. Recently, 
Chen et $a^{24}$ gave us a first glimpse by using RNA interference to silence Cdkl5 expression in rat cortical neurons. They demonstrated that Cdkl5 is essential for neuronal morphogenesis as its downregulation in cultured neurons inhibits neurite outgrowth and dendritic arborization whereas its over-expression elicits the opposite effect. At present it is not clear whether these findings recapitulate the situation in affected human brain. To answer this question, we have reprogrammed fibroblasts from patients carrying different CDKL5 mutations into iPSCs with the aim of establishing a human neuronal model for CDKL5-associated disease. We report here that female CDKL5mutated iPSCs maintain X-chromosome inactivation, have normal molecular karyotype indicating that they are isogenic, and we identify clones expressing either the wild-type or the mutant CDKL5 allele. We show that the cells can be differentiated into neurons and are thus suitable to model disease pathogenesis.

\section{METHODS}

\section{Fibroblasts reprogramming and hiPSCs culture}

Following informed consent signature, skin biopsies (about $3-4 \mathrm{~mm}^{3}$ ) were performed using the Punch Biopsy procedure. Fibroblasts were isolated and cultured with standard protocols. ${ }^{25}$ Fibroblasts at passage 2 or 3 were reprogrammed following the protocol by Hotta et $a 2^{26}$ that allows to enrich for reprogrammed cells. Briefly, fibroblasts were first infected with a lentiviral vector to stably introduce a reporter gene (EGFP) and a puromycin resistance gene under the control of an hESCs-specific promoter; parallel infection with a second lentivirus encoding the mouse mSlc7al receptor allowed the subsequent use of mouse-specific retroviruses for the expression of the reprogramming factors (OCT-4, SOX-2, c-MYC and KLF-4), reducing the risks of infection for operators. Seven days after retrovirus infection, fibroblasts were passed onto mitomycin-Cinactivated mouse embryo fibroblasts (feeders) and puromycin selection $(0.8 \mathrm{ug} / \mathrm{ml})$ (Invitrogen, Milan, Italy) was started on day 21 to selectively kill nonreprogrammed cells. Emerging iPSCs colonies were manually picked and expanded on feeders for some passages. Established clones that maintained a good hESCs-like morphology were moved to feeders-free culture conditions on Matrigel-coated dishes (BD Biosciences, Milano, Italy) in mTeSR1 medium (Stem Cell Technologies, Grenoble, France) with puromycin. From this point, cells were routinely passed by Collagenase IV treatment (Invitrogen).

\section{Clones characterization}

Genotyping. DNA from parental fibroblasts and iPSC clones was extracted with QIamp DNA mini kit (Qiagen, Milan, Italy) according to manufacturer's protocol. One hundred nanograms of DNA were PCR amplified using standard conditions and sequenced with the BigDye Terminator v1.1 Cycle Sequencing kit (Applied Biosystems, Milan, Italy) following manufacturer's protocol. Intronic primers flanking the exons harboring the mutations were used for both PCR and sequencing.

Immunostaining. Cells grown on coverslips were fixed with $4 \%$ paraformaldehyde in $1 \times \mathrm{PBS}$ for $10 \mathrm{~min}$ at $37^{\circ} \mathrm{C}$ and permeabilized with $1 \times \mathrm{PBS} / 0.01 \%$ Triton X-100 for $10 \mathrm{~min}$ at RT. Two hours incubation with blocking buffer (6\% NGS, 0.5\% BSA in PBS-T) at RT was performed. Subsequently, cells were incubated with appropriate dilutions of primary antibodies in blocking buffer $\mathrm{O} / \mathrm{N}$ at $4^{\circ} \mathrm{C}$. The next day, cells were washed three times with $1 \times \mathrm{PBS}-\mathrm{T}$ $(0.1 \%$ Tween 20 in $1 \times$ PBS $)$ and then incubated for $1 \mathrm{~h}$ with the secondary antibodies in blocking buffer. Finally, cells were washed three times with $1 \times$ PBS-T, stained with DAPI $1 \mu \mathrm{g} / \mathrm{ml}$ in $1 \times$ PBS for $10 \mathrm{~min}$. Coverslips were mounted with Mowiol and observed with a Axioscop 40FL (Zeiss, Milan, Italy) microscope connected to a computer. Images were merged and analyzed using ImageJ. Antibodies for the following hESCs-specific markers were used: NANOG (R\&D, Minneapolis, MN, USA), SSEA3 (Invitrogen), SSEA4 (Invitrogen), TRA-1-60 (Invitrogen), TRA-1-81 (Invitrogen).

In vitro differentiation via embryoid bodies formation. Cells were harvested by collagenase treatment and clumps were transferred on two wells of low-cluster 6-wells plates with embryoid bodies (EBs) medium (knockout
D-MEM supplemented with $10 \% \mathrm{FBS}, 2 \mathrm{~mm}$ L-Glutamine, $50 \mathrm{U} / \mathrm{ml}$ penicillin, $50 \mathrm{mg} / \mathrm{ml}$ streptomycin, $0.1 \mathrm{~mm}$ non-essential amino acids and $0.5 \mathrm{~mm}$ $\beta$-mercaptoethanol) and allowed to grow in suspension for 8 days. The resulting EBs were plated on collagen-coated coverslips $(0.05 \mathrm{mg} / \mathrm{ml}$ collagen acetic acid $0.1 \%$ in $\mathrm{H}_{2} \mathrm{O}$ ) and allowed to grow for another 8 days. The presence of differentiated cells of all three germ layers was tested by immunostaining (protocol described above) for lineage-specific markers: $\beta$-III-tubulin (Chemicon, Milan, Italy) for ectoderm, GATA-4 (Santa Cruz Biotechnologies, Heidelberg, Germany) for endoderm and smooth muscle actin (SMA; Invitrogen) for mesoderm.

Real-Time qPCR. Total RNA from iPS clones was isolated with Trizol reagent (Invitrogen). One microgram of total RNA was reverse transcribed with highcapacity cDNA reverse transcription kit (Applied Biosystems) according to manufacturer's instructions. Primer sets that allow the selective amplification of either the transgenes (OCT4, SOX2, KLF4 and C-MYC) or their endogenous counterparts were employed. Glyceraldehyde-3-phosphate dehydrogenase or actin were used as reference genes. PCR was carried out in single-plex reactions in a 96-well optical plate with FastStart SYBR Green Master Mix (Roche, Milan, Italy) on an ABI Prism 7700 Sequence Detection System (Applied Biosystems). Experiments were performed in triplicate in a final volume of $20 \mu \mathrm{l}$ with $5 \mathrm{ng}$ of cDNA and $150 \mathrm{~nm}$ of each primer, following SYBR Green protocol. Standard thermal cycling conditions were employed (Applied Biosystems): 2 min at $50^{\circ} \mathrm{C}$ and $10 \mathrm{~min}$ at $95^{\circ} \mathrm{C}$ followed by 40 cycles at $95^{\circ} \mathrm{C}$ for $15 \mathrm{~s}$ and $60^{\circ} \mathrm{C}$ for $1 \mathrm{~min}$. Results were analyzed using the comparative $\mathrm{Ct}$ method.

Molecular karyotyping by array-CGH. Array-CGH analysis was carried out on DNA extracted from clones at passage 15 or later using commercially available oligonucleotide microarrays with an estimated average spatial resolution of $\sim 45 \mathrm{~kb}$ (Human Genome CGH Microarray 44B Kit; Agilent Technologies, Milan, Italy) as previously reported (Sampieri K Cancer Sci 2009). Image analysis was carried out using CGH Analytics Software v. 3.4.40 (Agilent Technologies) with default settings. DNA from parental fibroblasts was also analyzed to identify constitutional CNVs.

\section{X-inactivation analysis}

The X-inactivation status was estimated using an assay based on a methylationsensitive HpaII restriction site located in the androgen receptor gene. ${ }^{27}$ This site is methylated on the inactive $\mathrm{X}$, and unmethylated on the active $\mathrm{X}$ chromosome. A polymorphic CAG repeat located within the amplified region is used to distinguish the two alleles. Genomic DNA from iPSCs and parental fibroblasts was digested with HpaII enzyme $\mathrm{O} / \mathrm{N}$ at $37^{\circ} \mathrm{C}$. For each sample two separate PCR reactions, on digested and undigested DNA, were performed using a fluorescent-dye-labelled primer set (Applied Biosystems). PCR products were run on an ABIPRISM GA3130 sequencer and analyzed with Gene Mapper software (Applied Biosystems). To estimate fragments length, FAM-labelled products were simultaneously compared with a LIZ size standard. PCR products from digested and undigested DNA were compared to estimate the $\mathrm{X}$-chromosome inactivation (XCI) status.

\section{Neuronal differentiation}

The experiment was performed on two clones from patient 1 (iPSC\#19 expressing mutant CDKL5 allele and iPSC \#20 expressing wild-type CDKL5) and a normal male control iPS clone derived from a newborn male BJ fibroblast line (cell line no CRL-2522) obtained from ATCC. ${ }^{26}$ Neuronal differentiation was performed following a protocol established for human ESCs. ${ }^{28,29}$ Briefly, cells were allowed to grow as EBs for 4 days in human ES medium (knockout DMEM supplemented with $15 \%$ KO serum replacement, $2 \mathrm{mM}$ L-glutamine, $50 \mathrm{U} / \mathrm{ml}$ penicillin, $50 \mathrm{mg} / \mathrm{ml}$ streptomycin, $0.1 \mathrm{~mm}$ non-essential amino acids and $0.5 \mathrm{~mm} \beta$-mercaptoethanol) and for 2 additional days in neuronal medium (DMEM/F12 supplemented with $1 \times \mathrm{N} 2,0.1 \mathrm{~mm}$ non-essential amino acids and $2 \mu \mathrm{g} / \mathrm{ml}$ Heparin). At day 6 , cells were plated onto laminin-coated plates and allowed to grow for 9 additional days. In this period neural precursor cells organized in neural rosettes emerged. The propensity of cells to differentiate toward the neuronal lineage was determined by calculating the percentage of plated EBs that formed neural rosettes. Rosettes were then manually lifted and allowed to grow as floating neurospheres for some days in neuronal medium 
plus $1 \times$ B27 supplement. Finally, neurospheres were plated onto poly- $N$ ornithine and laminin-coated slides or plates for final differentiation.

Based on data on human ESCs, the protocol is expected to originate mainly glutamatergic neurons. ${ }^{28,29}$ To confirm the identity of the obtained neurons, immunofluorescence was performed as described above using markers for both glutamatergic and GABAergic neurons: VGLUT1 (Invitrogen) and GAD65/67 (Chemicon). The generic neuronal markers $\beta$-III-tubulin or MAP2 (Abcam, Cambridge, UK) were used for neurons visualization. In addition, RT-PCR analysis was performed for the expression of markers of glutamatergic (VGLUT1, VGLUT2 and TBR1) and GABAergic (GAD67) neurons as well as glial cells (GFAP). Primer sequences are reported in Supplementary Table 1. Finally, in order to compare the efficiency of differentiation into glutamatergic and GABAergic neurons between different clones, real-time quantitative RTPCR experiments were performed using commercial TaqMan probes (VGLUT1 assay id: Hs00220404_ml; GAD67 assay id: Hs01065893 m1) on an ABI Prism 7700 Sequence Detection System. The glyceraldehyde-3-phosphate dehydrogenase gene was used as a reference (Applied Biosystems). Experiments were performed and analyzed as previously described. ${ }^{30}$ The Student's $t$-test with a significance level of $95 \%$ was used for the identification of statistically significant differences in expression levels among different clones.

\section{RESULTS}

\section{Patients selection}

Patient 1 (no. 1567) is a 2-year-old girl with a clinical diagnosis of early onset seizures variant of RTT who started presenting epilepsy not controlled by therapy at 1 month of age. At present she is not able to walk and she does not speak. Hand stereotypies, esophageal reflux, constipation, sleep disturbances are present. She also shows normal head circumference, poor visual contact and social interaction. Detailed clinical information has been already reported ${ }^{31}$ (Patient 1).

Patient 2 (no. 1559) is a 9-year-old boy with normal development in the first 16 months of life. At the age of 8 months, the first epileptic fits appeared and later on he showed developmental arrest and language regression. At 13 months he was able to say several words; however, at present he is only able to utter a few disyllabic words. He can walk without assistance and presently he shows four to six epileptic episodes a day without any clinical amelioration by pharmacological treatment. He also presents cold feet, constipation and normal head circumference. No hand stereotypies are referred. Detailed clinical information is reported in Elia et $a^{32}$ (Patient 2).

The two patients present different CDKL5 mutations (Figure 1a): patient 1 has a truncating mutation (p.Q347X) resulting in a protein lacking $2 / 3$ of its amino acids, including the domains responsible for the interaction with MeCP2 and DNMT1, and the nuclear export signal; patient 2 has a missense change (p.T288I) affecting a highly conserved amino acid inside the catalytic kinase domain. Patient fibroblasts were obtained from the 'Cell lines and DNA bank of Rett Syndrome and other X-linked mental retardation' biobank.

\section{Generation and Characterization of iPS cells}

Patient fibroblasts at low passage number were reprogrammed using a published protocol. ${ }^{14,26}$ Two weeks after infection with the reprogramming transgenes, the first morphological changes were visible, and compact iPSC-like colonies were identified 1-2 weeks later. At least 25 colonies for each patient were picked and expanded (Figure 1b). Three clones from patient 1 and five from patient 2 were selected for further characterization. Direct sequencing of the CDKL5 genomic region confirmed that iPSCs contained the expected mutation and thus were derived from the respective patient fibroblasts (data not shown). Standard tests were performed to confirm the fully reprogrammed status of the selected clones. All clones had a typical hESC-like morphology and expressed hESC-specific antigens such as NANOG, SSEA3, SSEA4, TRA-1-60 and TRA-1-81 (Figure 1c). Quantitative
RT-PCR experiments demonstrated that all but one clone silenced the four retroviral transgenes (OCT4, SOX2, KLF4, c-MYC) and reactivated the corresponding endogenous genes at levels comparable to those of hESCs (Figures $2 \mathrm{a}$ and $\mathrm{b}$ ). The clone derived from patient 2 that retained partial transgene expression was excluded from further analyses. In vitro differentiation via embryoid body formation confirmed the ability of the clones to spontaneously differentiate into cells of the three germ layers (Figures $2 \mathrm{~d}$ and e). Molecular karyotyping was performed by array-CGH to test whether the clones retained a normal karyotype. The same analysis was performed also on parental fibroblasts, to identify constitutive CNVs already present in the patients. All three clones deriving from patient 1 had a normal 46XX karyotype (Figure $2 \mathrm{c}$ and Supplementary Figure 1A). A polymorphic duplication in Xq22.2 was identified in the three clones, as well as in the parental fibroblasts (Supplementary Figure 1A). Of the four clones from patient 2 that passed previous characterization steps, a normal $46 \mathrm{XY}$ karyotype was found in three. Analysis of the fourth clone (iPSC\#52) revealed a trisomy of chromosome 8 (Supplementary Figure 1B) and this line was not studied further. These data indicate that no detectable de novo CNVs arose during reprogramming and that the cells are isogenic.

\section{$\mathrm{X}$-inactivation analysis}

As CDKL5 is an X-linked gene, we decided to test whether in female iPSCs X-inactivation was maintained or erased. To this aim we used the standard androgen-receptor gene test. ${ }^{27}$ The assay was initially performed on the three characterized clones and demonstrated that all had retained XCI and consistently showed only one active X chromosome (Figure $3 \mathrm{a}$ ). We thus extended the analysis to three additional clones derived from the same patient. In all three clones, the analysis demonstrated the presence of only one active $\mathrm{X}$ chromosome (Table 1). Interestingly, we identified clones deriving from the same patient but with different inactive X chromosomes (Figure 3a). These results suggested that they might express either the wild-type or the mutated CDKL5 allele. To confirm this hypothesis we sequenced CDKL5 mRNA (Figure 3b). As expected, parental fibroblasts showed expression of both alleles, consistent with random $\mathrm{X}$ inactivation. On the contrary, the iPS clones exclusively expressed only one allele; in accordance with results of XCI analysis, one clone expressed the wildtype CDKL5 allele, whereas the others expressed the mutated allele (Figure $3 \mathrm{~b}$ and data not shown). To confirm that this situation was stably maintained upon cell expansion in culture, XCI analysis was performed on cells at later passages for the two clones that were selected for further experiments (iPSC\#19 and \#20). The initial analysis had been performed on cells at passage 29 (clone \#19) and 21 (clone \#20). We thus analyzed cells at passages 32 and 41 for clone $\# 19$ and 23, and 32 for clone \#20. For both clones, cells at later passages retained XCI (Table 1).

\section{Neuronal differentiation}

To confirm that cells were suitable as an in vitro model to study the pathogenesis of CDKL5-related disorders, we induced the iPSCs toward a neuronal fate. ${ }^{28,29}$ In order to avoid confounding effects due to differences in genetic background, we decided to concentrate our attention on the two clones from patient 1 that are genotypically identical but differ for CDKL5 expression (iPSC\#19 expressing mutant CDKL5 allele and iPSC\#20 expressing wild-type CDKL5). $\mathrm{BJ}$-iPS clone derived from a normal male individual was included as a control for the differentiation process. Visual examination of cells all along the differentiation process suggested that our clones differentiated following the expected course (Figures $4 \mathrm{a}-\mathrm{f}$ ). Neural rosettes 
a
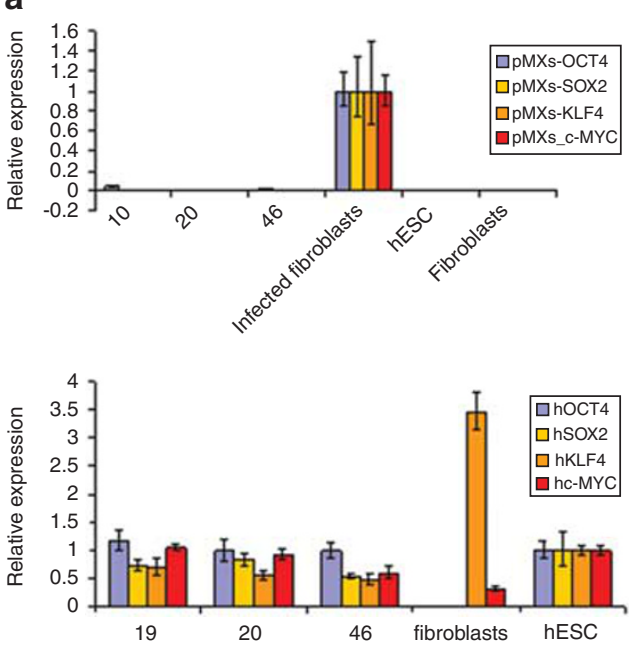

C

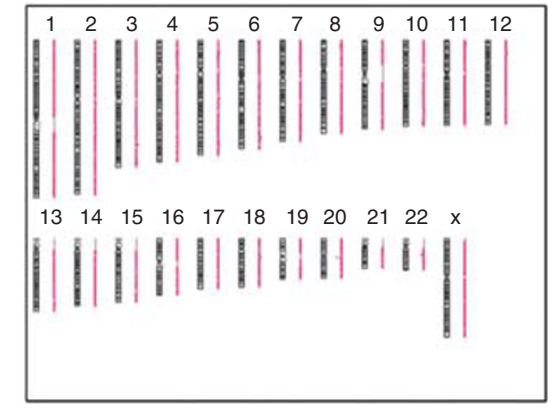

b
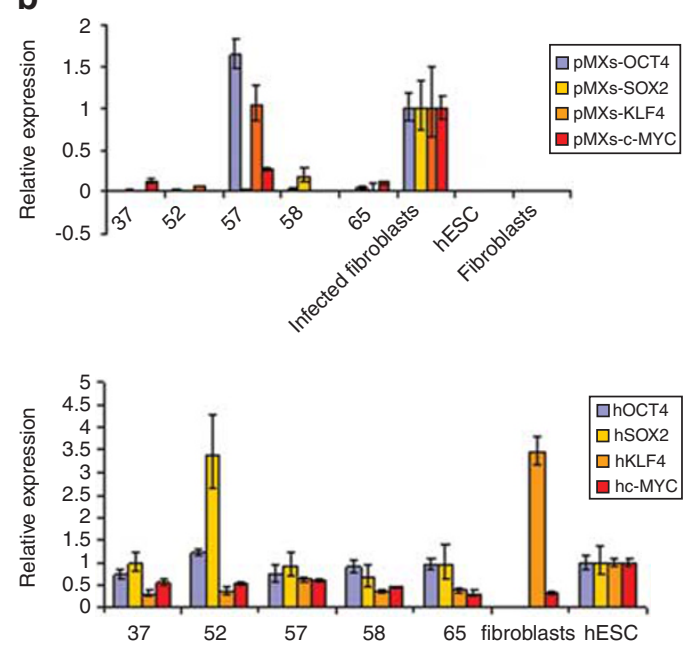

d

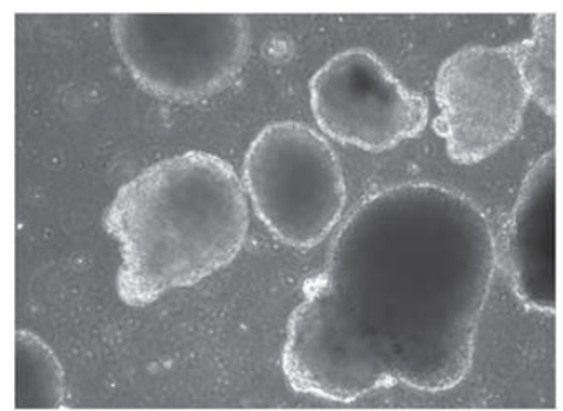

iPSC\#20
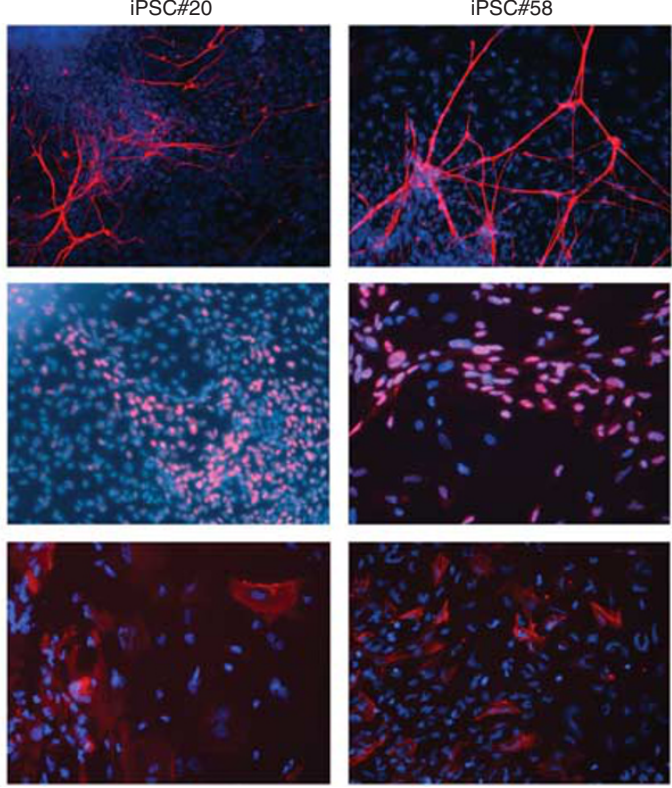
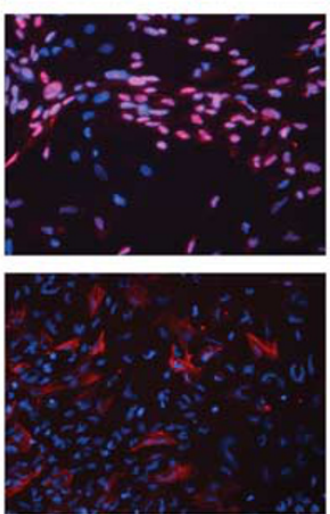

Figure 2 iPSCs characterization. (a, b). Summary of real-time RT-PCR experiments demonstrating that clones derived from patient 1 (a) and from patient 2 (b) have inactivated the four transgenes (upper panel in a, b) and reactivated the corresponding endogenous genes (lower panel). A human ESC line and freshly infected fibroblasts were used as positive controls for the expression of endogenous genes and transgenes, respectively. Parental fibroblasts were also analyzed. Clone \#57 from patient 2 maintained transgenes expression and it was thus excluded from further experiments. (c) Representative array CGH result from iPSC \#19 from patient 1 showing a normal karyotype. (d) Phase contrast image of EBs after 5 days of suspension culture. (e) Immunostaining of EBs after 16 days of differentiation shows staining for markers specific of all three germ layers: $\beta$-III-tubulin (ectoderm), GATA-4 (endoderm) and smooth muscle actin (SMA; mesoderm). Representative images of two clones from patient 1 (iPSC \#19 and iPSC \#20) and one clone from patient 2 (iPSC \#58) are shown. Images are at $\times 20$ magnification. 
a

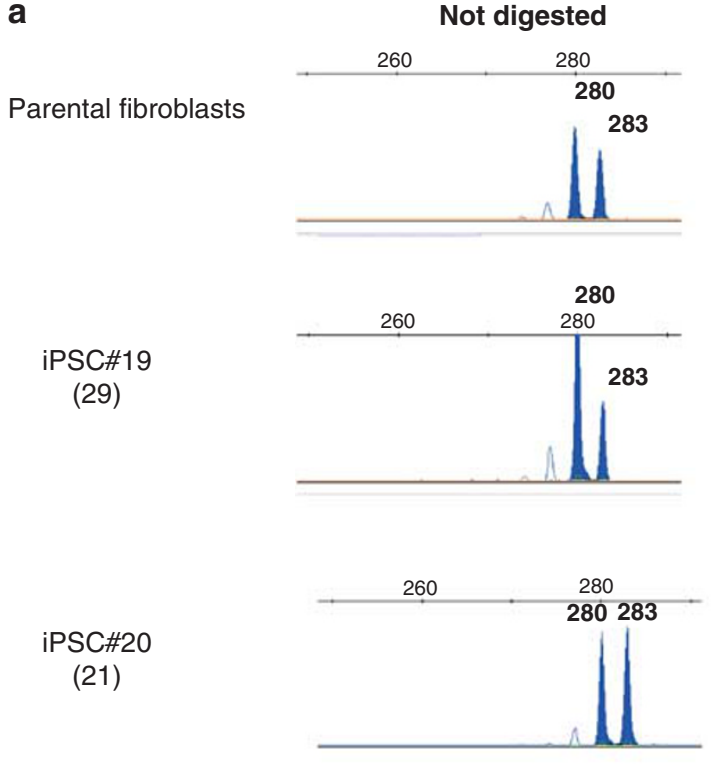

iPSC\#46

(25)

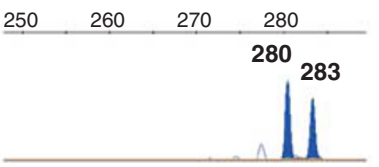

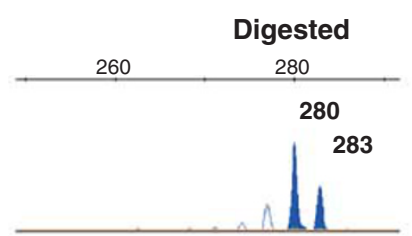
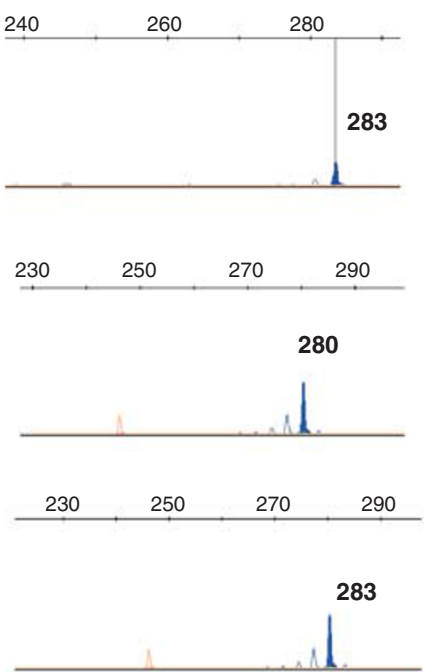

b

WT sequence

Mutated sequence

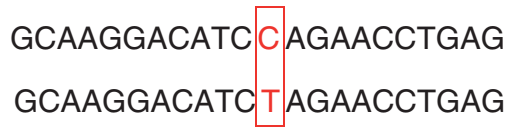

Parental fibroblasts

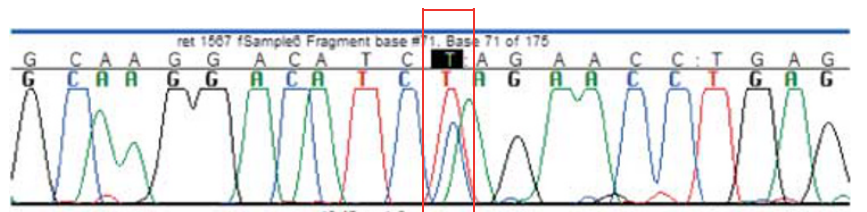

Clone\#19

(29)

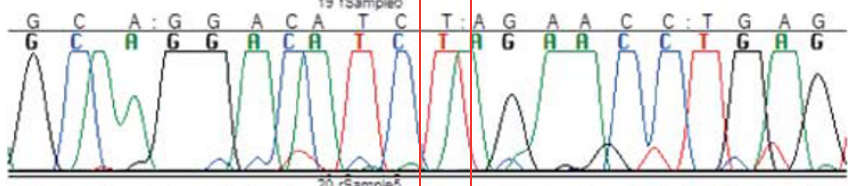

Clone\#20

(21)

Clone\#46

(25)
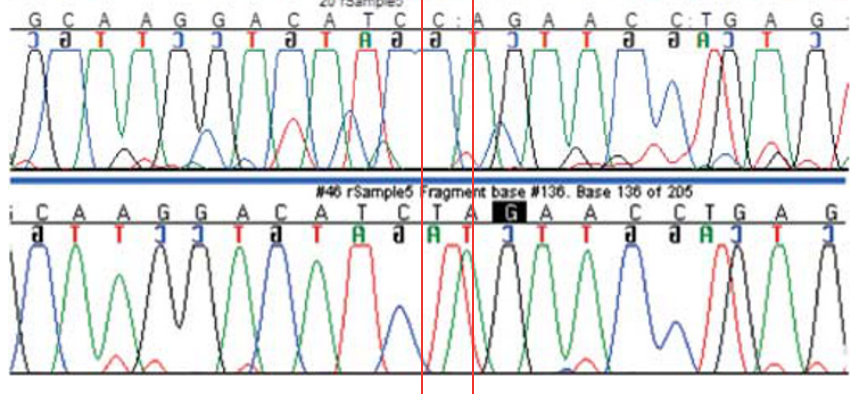

Figure 3 X-inactivation analysis. (a) In parental fibroblasts two alleles can be identified. PCR on digested DNA still result in two peaks, indicating a balanced XCI pattern. Undigested DNA from the three iPS clones (\#19, \#20 and \#46) present the same two peaks observed in parental fibroblasts. However, following digestion, each clone present only one peak, indicating a skewed pattern of $\mathrm{XCl}$. Comparison of alleles length demonstrates that the three clones inactivate different $X$ chromosomes. (b) Direct sequencing on cDNA from parental fibroblasts and iPSCs clones \#19, \#20 and \#46 from patient 1 . Wild-type and mutated sequences are shown at the top with the mutated nucleotide outlined in red. The chromatograms show that RNA isolated from parental fibroblasts presents both alleles whereas iPSCs clones express only one allele (mutated nucleotide outlined by a red rectangle). In particular, clones \#19 and \#46 express the mutated CDKL5 allele whereas clone \#20 expresses the wt (wild-type) allele. Passage number at the moment of DNA and RNA extraction is indicated in parenthesis below clone number. 
Table $1 \mathrm{XCl}$ analysis

\begin{tabular}{lclc}
\hline Sample & Passage no. & X-inactivation status & $\begin{array}{c}\text { Size of inactive } \\
\text { allelea }\end{array}$ \\
\hline iPSC \#19 & 32 & Skewed & 283 \\
iPSC \#19 & 41 & Skewed & 283 \\
iPSC \#20 & 23 & Skewed & 280 \\
iPSC \#20 & 32 & Skewed & 280 \\
iPSC \#33 & 13 & Skewed & 283 \\
iPSC \#48 & 16 & Skewed & 283 \\
iPSC \#60 & 24 & Skewed & 283 \\
\hline
\end{tabular}

Abbreviations: iPSC, induced pluripotent stem cell; XCl, X-chromosome inactivation. aThe inactive allele corresponds to the one that is amplified also after Hpall digestion due to methylation of the enzyme-recognition sequence.

consisting of one or more layers of columnar cells arranged in a tubular structure were visible in all clones a few days after EBs seeding (Figure $4 \mathrm{a}$ ). Shortly after plating the neurospheres for final differentiation, numerous cell processes started to emerge from the spheres giving them a star-like appearance (Figure $4 \mathrm{~b}$ ). When allowed to differentiate further, cell processes formed bundles of fibers and cells could be seen migrating away from the spheres (Figures $4 \mathrm{c}$ and d). At this stage, MAP2-positive neurons could be identified for all clones (Figures $4 \mathrm{e}$ and $\mathrm{f}$ ). Based on data on human ES, the protocol was expected to originate mainly glutamatergic neurons. ${ }^{28,29}$ To determine whether this was indeed the case, cells were differentiated for 10 weeks and mature neurons were analyzed. RT-PCR with primers for glutamatergic (VGLUT1, VGLUT2 and TBR1) and GABAergic (GAD67) neurons demonstrated that the cultures contained both neuronal types (Figure 4j). GFAP-positive glial cells were also present (Figure 4j). This result was confirmed by immunostaining for VGLUT1 and GAD65/ 67. In particular, our data indicate that, as expected, the majority of the resulting neurons are glutamatergic but GABAergic neurons are also present (Figures $4 \mathrm{~g}$ and $\mathrm{h}$ ). Mature cultures also contained many non-neuronal cells that were negative for both MAP2 and $\beta$-IIItubulin staining (Figure 4i).

In order to obtain a first estimate of the ability of our clones to differentiate toward a neuronal fate, the percentage of seeded EBs that formed clearly identifiable rosette structures on day 15 was calculated (Figure 4k). The analysis was performed on two independent experiments, with comparable results. The clones formed rosettes with efficiency ranging from $37 \%$ in the BJ-iPS to $47 \%$ in clone \#19. Interestingly, the two clones derived from patient 1 (iPSC \#19 and \#20) displayed very similar efficiencies (47\% and $46 \%$, respectively) (Figure $4 \mathrm{k}$ ). We then decided to evaluate whether the different clones formed glutamatergic and GABAergic neurons with the same efficiency. No gross differences were visible but the high density of the neuronal network arising in culture did not allow us to calculate the percentage of cells in the two populations from immunofluorescence images. As a consequence, to evaluate differences in the potential of single clones to differentiate into glutamatergic or GABAergic neurons, real-time quantitative RT-PCR was performed on RNA extracted from 10-week-old neurons using probes for VGLUT1 or GAD67, respectively (Figure 41). Our results showed that the three analyzed clones differed significantly from each other for VGLUT1 expression, with the highest expression in iPSC \#20. GAD67 expression was comparable between iPSC\#19 and the normal control clone, whereas iPCS \#20 again showed significantly higher expression.

\section{DISCUSSION}

RTT is one of the most common causes of mental retardation in females. In spite of intense research efforts, clarification of the molecular mechanisms of RTT, as well as all other neurologic disorders, has been hampered by the lack of satisfactory human in vitro cellular models. Mouse models represent a useful alternative to mimic human monogenic neurological disorders and they have allowed important insights into disease mechanisms of RTT due to MECP2 mutations. However, mouse models often do not faithfully recapitulate human conditions and they do not take into account the influence of genetic background on disease phenotype. In this respect, an unprecedented opportunity for the creation of patient- and disease-specific human cellular models is represented by iPSCs. ${ }^{15}$ Very recently iPSCs technology has been applied to model RTT starting from fibroblasts of MECP2-mutated patients. ${ }^{18}$ MECP2 is the most commonly mutated gene in classic RTT, and it has thus been the most extensively studied. Less is known about CDKL5, the gene associated to the early onset seizures variant of RTT and to other forms of X-linked intellectual disabilities. ${ }^{19,20}$ In order to clarify the role of CDKL5 in human affected neurons, we used iPSC technology to establish a human cellular model that recapitulates CDKL5-related disorders. To this aim, we reprogrammed fibroblasts from a hemizygous male with a missense mutation affecting an highly conserved residue inside the catalytic kinase domain, and a heterozygous female with a stop mutation downstream of the kinase domain predicted to result in a truncated protein lacking $2 / 3$ of its amino acids. ${ }^{31,32}$ Fibroblasts from both patients were successfully reprogrammed. iPSCs lacking a functional CDKL5 protein (those derived from the hemizygous male patient and those deriving from the female but expressing only mutant CDKL5) did not show obvious differences in proliferation with respect to the clone expressing wild-type allele or to BJ-iPS and could be easily passaged several times (at least 25 passages) without any apparent change.

Recent studies report that the reprogramming process is associated with accumulation of de novo CNVs, especially in early passage iPSCs. ${ }^{33,34}$ We thus decided to perform array-CGH analysis in our pluripotent clones in order to more accurately check for the presence of imbalances deriving from the reprogramming process. Among the seven clones that we analyzed (three from patient 1 and 4 from patient 2 ), only one presented a trisomy of chromosome 8 and was not studied further, while the others did not show any obvious alteration (Figure 2c and Supplementary Figure 1). Although our CNV detection method is less sensitive than previous studies $(75 \mathrm{~kb}$ compared with $30 \mathrm{~kb}$ ), our results from clones at passages higher than 15 are consistent with the report by Hussein et al that de novo CNVs are selected against during expansion in culture. Thus, the iPS cell clones we derived are isogenic and display a normal molecular karyotype at this resolution.

In order to allow dosage-compensation for genes on the $\mathrm{X}$ chromosome between males and females, in mammalian female cells one of the two X chromosomes is silenced early during development through a mechanism defined as XCI. ${ }^{35}$ In accordance with their derivation from the inner cell mass of the blastocyst, at a pre-XCI stage, female mouse ESCs carry two active X chromosomes that undergo random XCI upon differentiation. ${ }^{36}$ The same situation is observed in mouse iPSCs. ${ }^{37}$ Human ESCs reveal a heterogeneous status: most undifferentiated hESC lines carry an inactive $\mathrm{X}$ chromosome, but some sublines containing two active $\mathrm{X}$ chromosomes can be identified. ${ }^{38}$ XCI status in human iPSCs is still controversial. In fact, it has been demonstrated that Duchenne muscular dystrophy and MECP2-null iPS cells retain an inactive $\mathrm{X}$ chromosome in a clonal pattern with one 

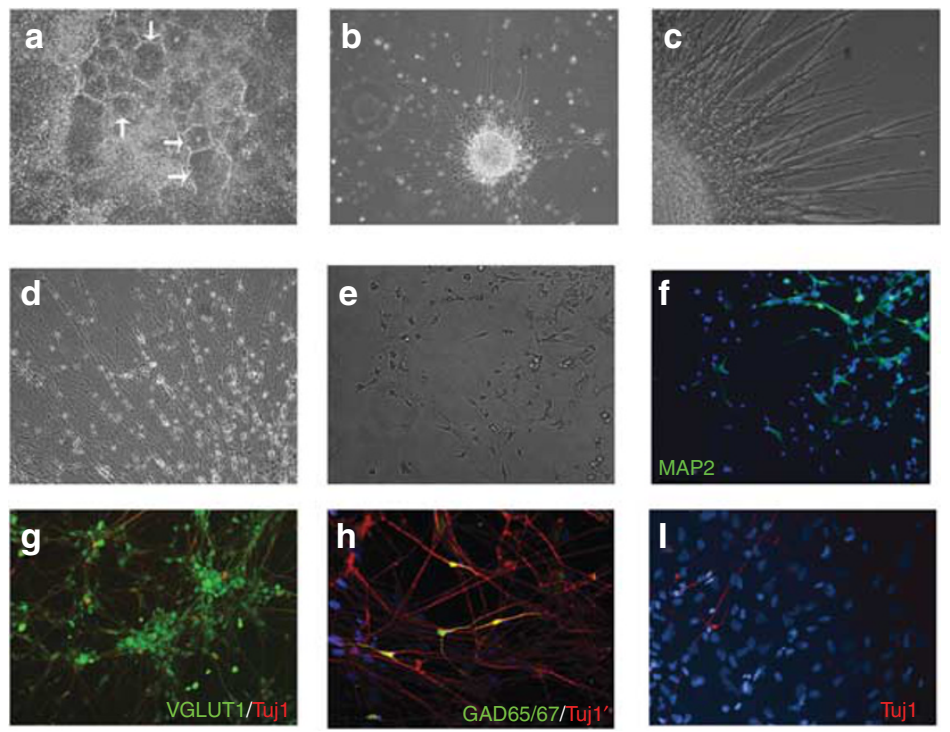

j

k
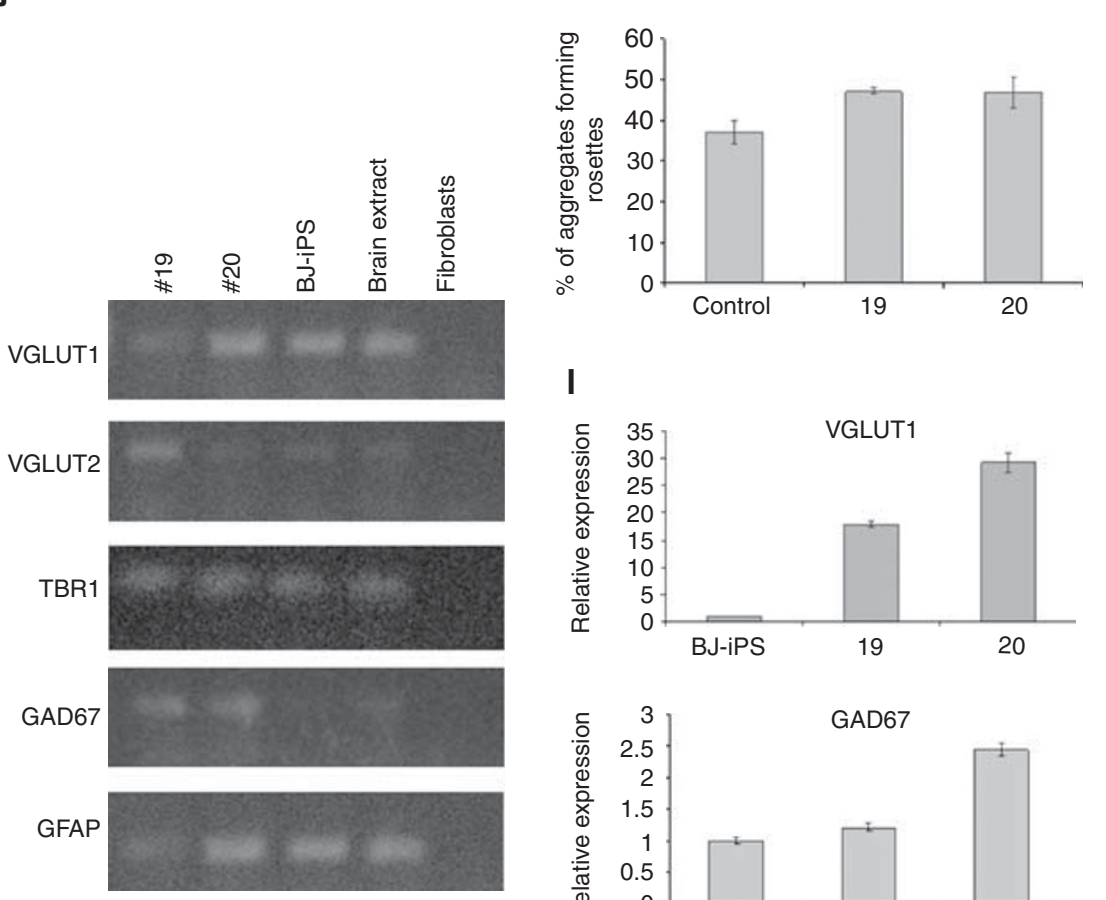

I
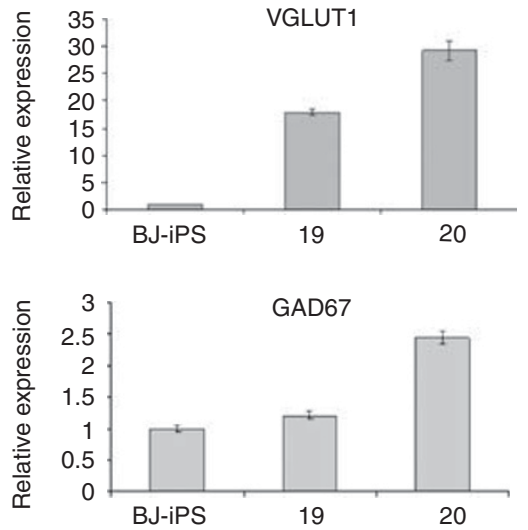

Figure 4 CDKL5-mutated iPSCs differentiate into neurons. iPSCs were induced to differentiate into neurons following a published protocol. (a) Neural rosettes (arrows) consisting of columnar cells arranged in a tubular structure were visible a few days after EBs seeding. (b) Shortly after neurospheres plating, numerous cell processes started to emerge from the spheres giving them a star-like appearance. (c, d) When cells were allowed to differentiate further, cell processes formed bundles of fibers (c) and cells could be seen migrating away from the spheres (d). (e, f) At this stage MAP2-positive neurons (green in f) could be identified by immunofluorescence. (g, h) After 10 weeks of differentiation the majority of $\beta$-III-tubulin-positive neurons (Tuj1, red) were also positive for VGLUT1 (green in g), but cells positive for the GABAergic marker GAD65/67 could be also identified (green in $\mathbf{h}$ ). (i) In addition to neurons, many $\beta$-IIItubulin-negative non-neuronal cells were present in our cultures, as evidenced by DAPI-positive nuclei (blue). Images are at $\times 20$ magnification. Blue staining=DAPI. (j) RT-PCR analysis on RNA isolated from 10-week-old neuronal cultures demonstrates that glutamatergic (VGLUT1, VGLUT2, TBR1) and GABAergic (GAD67) neurons, and glial cells (GFAP) are present in our cultures. RNA isolated from parental fibroblasts was used as negative control; a commercial RNA from human total brain was used as positive control. (k) The ability of our cells to differentiate toward a neuronal fate was estimated as the percentage of plated EBs that formed rosettes on day 15. Histograms represent the mean of two independent experiments. Error bars represent standard deviation. (I) Results of quantitative real-time RT-PCR on neuronal cultures show a high variability of expression of both VGLUT1 (upper panel; $P<0.0001$ ) and GAD67 (lower panel; $P<0.0001$ between BJ-iPS and \#20, and between \#19 and \#20; $P<0.05$ between BJ-iPS and \#19) in the three clones. Expression in neurons derived from the normal control clone was arbitrarily set as 1 . Error bars represent standard deviation from three replicates of each sample. 
of the two X chromosomes exclusively inactivated in all the cells of a single clone. ${ }^{39,40}$ However, Marchetto et al ${ }^{18}$ recently reported that $\mathrm{X}$ inactivation can also be erased during reprogramming of hiPSCs and it is subsequently restored upon differentiation. Considering this controversy and as CDKL5 is an X-linked gene that when mutated causes RTT like MECP2, we tested XCI in our female iPSCs clones from patient 1 . In accordance with the data reported by Tchieu et al and Cheung et al our clones present XCI. Interestingly, while parental fibroblasts show random XCI, each clone exclusively expresses one of the two X chromosomes (Figure 3 and Table 1). Moreover, analysis of the same clones at different passage numbers indicated that they stably maintained exclusive expression of the same $\mathrm{X}$ chromosome upon expansion in culture (Table 1). The clonal nature of XCI in our clones and in those reported by Tchieu ${ }^{39}$ and by Cheung ${ }^{40}$ confirm the hypothesis that in human cells XCI is usually not erased during reprogramming, but each iPSC clone maintains the same inactive $\mathrm{X}$ chromosome as the original reprogrammed fibroblast from which it was derived. ${ }^{39}$ Apart from the general relevance to our understanding of the reprogramming process, the finding that hiPSCs retain XCI has important implications also for the application of these cells for in vitro modelling of X-linked disorders. In fact, it implies that from female fibroblasts with random XCI, it is possible to obtain iPSCs expressing either the mutant or wild-type allele of an X-linked gene. ${ }^{39}$ This is in fact what we found for hiPSCs derived from the female patient (Figure 3). The clone expressing the WT allele is genetically identical to those expressing the mutated one; it thus represents the ideal isogenic control to test the effects of a mutation without the potentially confounding effect of genetic background.

To test whether our CDKL5-mutated hiPSCs could be used to model disease pathogenesis in vitro, we differentiated them into neurons following a protocol established for hESCs. ${ }^{28,29,41}$ The characterization of the neuronal differentiation process indicates that the cells can be differentiated into neurons and that the differentiation follows the stages observed in hESCs (Figures $4 \mathrm{a}-\mathrm{f}$ ). ${ }^{41}$ The clones show a variable but reproducible efficiency of differentiation toward the neuronal lineage, as already reported for iPSCs (Figure $4 \mathrm{k}$ ). ${ }^{42}$ Interestingly, the two clones derived from patient 1 but expressing different CDKL5 alleles (iPSC\#19 and iPSC\#20) have similar efficiencies, suggesting that the absence of a functional CDKL5 protein does not affect the initial commitment toward a neuronal fate.

Based on data on hESCs, we expect the resulting cells to be mainly excitatory glutamatergic neurons. ${ }^{29,41}$ Immunofluorescence analysis with specific markers indicates that this is indeed the case, even though GABAergic neurons can be also identified (Figures $4 \mathrm{~g}$ and $\mathrm{h}$ ). The very dense network of cells in mature cultures did not allow us to directly estimate the relative percentages of neurons in the two populations. We thus decided to compare the ability of different clones to give rise to glutamatergic and GABAergic neurons by estimating the amount of VGLUT1 or GAD67 mRNA in mature cultures. We found very different expression levels for the two markers between the three analyzed clones (Figure 41). In particular, for VGLUT1 the difference is statistically significant both between iPSC\#19, expressing mutant CDKL5, and the other two clones, and between these last two clones that both express wild-type CDKL5 (iPSC \#20 and the control clone). Moreover, the difference is higher between these last two clones than with iPSC\#19. This suggested that the observed difference is not a specific consequence of CDKL5 absence but rather reflects clone-to-clone variability. For GAD67, iPSC\#19 and the control clone present similar expression, but iPSC\#20 has a statistically higher expression. This high variability was unexpected, as the evaluation of the ability of the clones to form neural rosettes did not show dramatic differences between the three clones. To try to understand the reasons for these differences we thus examined our immunofluorescence slides. The mature cultures contained many non-neuronal cells that were negative for both MAP2 and $\beta$-III-tubulin staining. This is in accordance with other reports that indicate that a percentage of non-neuronal cells remain in the cultures. ${ }^{43,44}$ In particular, although an accurate quantitation could not be performed, cultures deriving from the BJ-iPS clone seem to have a higher number of non-neuronal cells compared with the other two clones. As this clone showed lowest expression of both VGLUT1 and GAD67, this finding suggests that heterogeneity of the neuronal cultures might be explained at least in part by the variable presence of non-neuronal cells. An alternative hypothesis is the presence of variable percentages of other neuronal types. Although we cannot presently confirm or exclude this hypothesis, the fact that the cells follow the expected differentiation course point to the presence of non-neuronal cells as the factor responsible for the apparent variability of VGLUT1 and GAD67 expression. It will be thus essential to develop accurate methods to enrich for neuronal precursors in order to have pure populations amenable to quantitative analyses. ${ }^{43}$

The protocol we employed gives rise mainly to excitatory glutamatergic neurons. ${ }^{29,41}$ However, Li et al ${ }^{29}$ reported that neural precursors can be redirected to form inhibitory GABAergic neurons by inhibition of Wnt pathway and/or activation of SHH. This seems particularly relevant for the modelling of CDKL5-related disease. In fact, glutamatergic excitatory neurotransmission has been reported to be altered in RTT due to MECP2 mutations. ${ }^{13}$ Considering the interaction between CDKL5 and MeCP2 and their common involvement in RTT, it is conceivable that CDKL5 absence might affect, at least in part, the same neuronal circuits. If this is the case, we could expect our cells to show a phenotype similar to that recently reported for MECP2-mutated hiPSCs-derived neurons. ${ }^{18}$ However, in mouse brain, CDKL5 is expressed at high levels also in GABAergic neurons. ${ }^{20}$ It will be thus interesting to differentiate our hiPSCs into both glutamatergic and GABAergic neurons in order to check whether a phenotype can be observed in both neuronal populations or whether CDKL5 absence specifically affects one neuronal type.

In conclusion, we report here the generation of iPSCs from two patients with CDKL5 mutations. We demonstrated that the female CDKL5-mutated iPSCs maintain X-chromosome inactivation, and we could identify clones expressing either the wild-type or the mutant CDKL5 allele. Neuronal differentiation experiments indicate that the cells can be differentiated into neurons and are thus suitable to model disease pathogenesis. However, expression analyses on the mature cultures suggest that methods to selectively isolate neuronal precursors are necessary if we want to perform accurate quantitative comparisons between the cells derived from different clones. The identification of a clone from the female patient expressing exclusively the wild-type CDKL5 allele represents a very exciting result, as this clone potentially represents the ideal isogenic control, genetically identical to those derived from the same patient but expressing the mutant allele, to study the effect of the mutation without 'perturbations' related to differences in genetic background.

\section{CONFLICT OF INTEREST}

The authors declare no conflict of interest.

\section{ACKNOWLEDGEMENTS}

The authors are grateful to Peter Pasceri and Tadeo Thompson for technical assistance. We acknowledge 'Cell Lines and DNA Bank of Rett syndrome and X-linked mental retardation’ (Medical Genetics-Siena) - Telethon Genetic 
Biobank Network (Project No. GTB07001C to AR), a Canadian Institutes of Health Research grant (MOP-102649 to JE), a Natural Sciences and Engineering Research Council of Canada Postgraduate Scholarship Doctoral Award (to AYLC), Telethon grant GGP09117 and a grant by A.I.R. (Associazione Italiana Rett) (to AR).

1 Chahrour M, Zoghbi HY: The story of Rett syndrome: from clinic to neurobiology. Neuron 2007; 56: 422-437.

2 Hagberg BA, Skjeldal $\mathrm{OH}$ : Rett variants: a suggested model for inclusion criteria Pediatr Neurol 1994; 11: 5-11.

3 Rolando S: Rett syndrome: report of eight cases. Brain Dev 1985; 7: 290-296.

4 Hanefeld F: The clinical pattern of the Rett syndrome. Brain Dev 1985; 7: 320-325.

5 Zappella M: The preserved speech variant of the Rett complex: a report of 8 cases. Eur Child Adolesc Psychiatry 1997; 6: 23-25.

6 Scala E, Ariani F, Mari F et al: CDKL5/STK9 is mutated in Rett syndrome variant with infantile spasms. J Med Genet 2005; 42: 103-107.

7 Ariani F, Hayek G, Rondinella D et al: FOXG1 is responsible for the congenital variant of Rett syndrome. Am J Hum Genet 2008; 83: 89-93.

8 Matijevic T, Knezevic J, Slavica M, Pavelic J: Rett syndrome: from the gene to the disease. Eur Neurol 2009; 61: 3-10.

9 Chahrour M, Jung SY, Shaw C et al: MeCP2, a key contributor to neurological disease, activates and represses transcription. Science 2008; 320: 1224-1229.

10 Guy J, Hendrich B, Holmes M, Martin JE, Bird A: A mouse Mecp2-null mutation causes neurological symptoms that mimic Rett syndrome. Nat Genet 2001; 27: 322-326.

11 Chen RZ, Akbarian S, Tudor M, Jaenisch R: Deficiency of methyl-CpG binding protein-2 in CNS neurons results in a Rett-like phenotype in mice. Nat Genet 2001; 27: 327-331.

12 Shahbazian M, Young J, Yuva-Paylor L et al: Mice with truncated MeCP2 recapitulate many Rett syndrome features and display hyperacetylation of histone H3. Neuron 2002; 35: 234-254.

13 Chao HT, Zoghbi HY, Rosenmund C: MeCP2 controls excitatory synaptic strength by regulating glutamatergic synapse number. Neuron 2007; 56: 58-65.

14 Takahashi K, Tanabe K, Ohnuki M et al: Induction of pluripotent stem cells from adult human fibroblasts by defined factors. Cell 2007; 131: 861-872.

15 Marchetto MC, Winner B, Gage FH: Pluripotent stem cells in neurodegenerative and neurodevelopmental diseases. Hum Mol Genet 2010; 19: R71-R76.

16 Chamberlain SJ, Chen PF, Ng KY et al: Induced pluripotent stem cell models of the genomic imprinting disorders Angelman and Prader-Willi syndromes. Proc Natl Acad Sci USA 2010; 107: 17668-17673.

17 Urbach A, Bar-Nur O, Daley GQ, Benvenisty N: Differential modeling of fragile $X$ syndrome by human embryonic stem cells and induced pluripotent stem cells. Cell Stem Cell 2010; 6: 407-411.

18 Marchetto MC, Carromeu C, Acab A et al: A model for neural development and treatment of Rett syndrome using human induced pluripotent stem cells. Cell 2010; 143: 527-539.

19 Mari F, Azimonti S, Bertani I et al: CDKL5 belongs to the same molecular pathway of MeCP2 and it is responsible for the early-onset seizure variant of Rett syndrome. Hum Mol Genet 2005; 14: 1935-1946.

20 Rusconi L, Salvatoni L, Giudici L et al: CDKL5 expression is modulated during neuronal development and its subcellular distribution is tightly regulated by the $\mathrm{C}$-terminal tail. J Biol Chem 2008; 283: 30101-30111.

21 Bertani I, Rusconi L, Bolognese F et al: Functional consequences of mutations in CDKL5, an X-linked gene involved in infantile spasms and mental retardation. J Biol Chem 2006; 281: 32048-32056.
22 Carouge D, Host L, Aunis D, Zwiller J, Anglard P: CDKL5 is a brain MeCP2 target gene regulated by DNA methylation. Neurobiol Dis 2010; 38: 414-424.

23 Kameshita I, Sekiguchi M, Hamasaki D et al: Cyclin-dependent kinase-like 5 binds and phosphorylates DNA methyltransferase 1. Biochem Biophys Res Commun 2008; 377 : 1162-1167.

24 Chen Q, Zhu YC, Yu J et al: CDKL5, a protein associated with Rett syndrome, regulates neuronal morphogenesis via Rac1 signaling. J Neurosci 2010; 30: 12777-12786.

25 Dracopoli N, Haines J, Korf B, Morton C, Seidman C, Smith D: Current Protocols in Human Genetics. NY: John Wiley \& Son, Inc., 2000.

26 Hotta A, Cheung AY, Farra N, et al: Isolation of human iPS cells using EOS lentiviral vectors to select for pluripotency. Nat Methods 2009; 6: 370-376.

27 Allen RC, Zoghbi HY, Moseley AB, Rosenblatt HM, Belmont JW: Methylation of $\mathrm{Hpall}$ and Hhal sites near the polymorphic CAG repeat in the human adrogen-recetor gene correlates with X chromosome inactivation. Am J Hum Genet 1992; 51: 1229-1239.

28 Pankratz MT, Li XJ, Lavaute TM, Lyons EA, Chen X, Zhang SC: Directed neural differentiation of human embryonic stem cells via an obligated primitive anterior stage. Stem Cells 2007; 25: 1511-1520.

29 Li XJ, Zhang X, Johnson MA, Wang ZB, Lavaute T, Zhang SC: Coordination of sonic hedgehog and Wnt signaling determines ventral and dorsal telencephalic neuron types from human embryonic stem cells. Development 2009; 136: 4055-4063.

30 Meloni I, Parri V, De Filippis R et al: The XLMR gene ACSL4 plays a role in dendritic spine architecture. Neuroscience 2009; 159: 657-669.

31 Artuso R: Early-onset seizure variant of Rett syndrome: definition of the clinical diagnostic criteria. Brain Dev 2010; 32: 17-24.

32 Elia M, Falco M, Ferri R et al: CDKL5 mutations in boys with severe encephalopathy and early-onset intractable epilepsy. Neurology 2008; 71: 997-999.

33 Laurent C: Dynamic changes in the copy number of pluripotency and cell proliferation genes in human ESCs and IPSCs during reprogramming and time in culture. Cell Stem Cell 2011; 8: 106-118.

34 Hussein M: Copy number variation and selection during reprogramming to pluripotency. Nature 2011; 471: 58-62.

35 Goto T, Monk M: Regulation of X-chromosome inactivation in development in mice and humans. Microbiol Mol Biol Rev 1998; 62: 362-378.

36 Nichols J, Smith A: Naive and primed pluripotent states. Cell Stem Cell 2009; 4: 487-492.

37 Maherali N, Sridharan R, Xie W et al: Directly reprogrammed fibroblasts show global epigenetic remodeling and widespread tissue contribution. Cell Stem Cell 2007; 1: 55-70.

38 Hall LL, Byron M, Butler J et al: X-inactivation reveals epigenetic anomalies in most hESC but identifies sublines that initiate as expected. J Cell Physiol 2008; 216: 445-452.

39 Tchieu J, Kuoy E, Chin MH et al: Female human iPSCs retain an inactive $\mathrm{X}$ chromosome. Cell Stem Cell 2010; 7: 329-342.

40 Cheung AY: Isolation of MECP2-null Rett Syndrome patient hiPS cells and isogenic controls through X-chromosome inactivation. Hum Mol Genet 2011; 20: 2103-2115.

41 Zhang SC, Wernig M, Duncan ID, Brustle O, Thomson JA: In vitro differentiation of transplantable neural precursors from human embryonic stem cells. Nat Biotechnol 2001; 19: 1129-1133.

$42 \mathrm{Hu}$ BY, Weick JP, Yu J et al: Neural differentiation of human induced pluripotent stem cells follows developmental principles but with variable potency. Proc Natl Acad Sci USA 2010; 107: 4335-4340.

43 Yuan S: Cell-surface marker signatures for the isolation of neural stem cells, glia and neurons derived from human pluripotent stem cells. PLOS ONE 2011; 6: e17540.

44 Carson CT, Aigner S, Gage FH: Stem cells: the good, bad and barely in control. Nat Med 2006; 12: 1237-1238.

Supplementary Information accompanies the paper on European Journal of Human Genetics website (http://www.nature.com/ejhg) 